\title{
Accuracy of dynamic contrast-enhanced magnetic resonance imaging in the diagnosis of prostate cancer: systematic review and meta-analysis
}

\author{
Zhiqiang Chen ${ }^{1,2, *}$, Yi Zheng ${ }^{2, *}$, Guanghai $\mathrm{Ji}^{2}$, Xinxin Liü ${ }^{3}$, Peng $\mathrm{Li}^{2}$, Lei Cai ${ }^{2}$, Yulin \\ Guo $^{2}$ and Jian Yang ${ }^{1}$ \\ ${ }^{1}$ Department of Radiology, The First Affiliated Hospital of Xi'an Jiaotong University, Xi'an 710061, Shaanxi, China \\ ${ }^{2}$ Radiology Department of The General Hospital, Ningxia Medical University, Yinchuan 750004, Ningxia, China \\ ${ }^{3}$ Department of Diagnostic Imaging, Honghui Hospital, Health Science Center of Xi'an Jiaotong University, Xi'an 750004, \\ Shaanxi, China \\ "These authors contributed equally to this work
}

Correspondence to: Jian Yang, email: yj1118@mail.xjtu.edu.cn

Keywords: diagnostic imaging, diagnostic techniques, early detection of cancer, magnetic resonance imaging, male

Received: March 03, 2017 Accepted: August 04, 2017 Published: August 17, 2017

Copyright: Chen et al. This is an open-access article distributed under the terms of the Creative Commons Attribution License 3.0 (CC BY 3.0 ), which permits unrestricted use, distribution, and reproduction in any medium, provided the original author and source are credited.

\section{ABSTRACT}

The goals of this meta-analysis were to assess the effectiveness of dynamic contrast-enhanced magnetic resonance imaging (DCE-MRI) in patients with prostate carcinoma (PCa) and to explore the risk profiles with the highest benefit. Systematic electronic searched were conducted in database. We used patient-based and biopsybased pooled weighted estimates of the sensitivity, specificity, and a summary receiver operating characteristic (SROC) curve for assessing the diagnostic performance of DCE. We performed direct and indirect comparisons of DCE and other methods of imaging. A total of $\mathbf{2 6}$ articles met the inclusion criteria for the analysis. DCE-MRI pooled sensitivity was 0.53 (95\% CI 0.39 to 0.67$)$, with a specificity of 0.88 (95\% CI 0.83 to 0.92 ) on whole gland. The peripheral zone pooled sensitivity was 0.70 ( $95 \%$ CI 0.46 to 0.86 ), with a specificity of 0.88 (95\% CI 0.76 to 0.94 ). Compared with T2-weighted imaging (T2WI), DCE was statistically superior to T2. In conclusion, DCE had relatively high specificity in detecting PCa but relatively low sensitivity as a complementary functional method. DCE-MRI might help clinicians exclude cases of normal tissue and serve as an adjunct to conventional imaging when seeking to identify tumor foci in patients with $\mathrm{PCa}$.

\section{INTRODUCTION}

Prostate cancer is the second most common form of cancer in males worldwide [1], and the growing elderly population has led to an increase in the number of estimated new prostate carcinoma (PCa) cases. PCa accounted for the highest number of estimated new cases of cancer and the second highest number due to cancer deaths of males in the United States in 2015, with totals of 220,800 and 27,540,respectively [2]. In the developed regions of East Asia (i.e., Korea, Japan, and the Taiwan and Shanghai regions of China), $\mathrm{PCa}$ is the fifth most common cancer as well as the most common genitourinary cancer, with approximately $9 \%(82,691)$ cancer cases diagnosed with $\mathrm{PCa}(8.2 / 100,000)$ [3]. By 2030, the global burden of $\mathrm{PCa}$ is expected to increase by 1.7 million new cases, and 499,000 deaths will occur because of population growth and aging worldwide [4].

PCa can initially develop slowly and remain limited to the prostate gland. However, some types develop quickly and become aggressive. Delays in the diagnosis of PCa reduce the likelihood of treating $\mathrm{PCa}$ in its early stages. Onwukamuche et al. showed that doctors only obtain a $36.4 \%$ sensitivity level with regard to the diagnosis of $\mathrm{PCa}$ [5], especially among patients who are in the early stages of this disease and exhibit no signs of illness. All available diagnostic tools for early detection, such as digital rectal examination (DRE), serum prostate-specific 
antigen (PSA; a nonspecific blood test) and transrectal ultrasound (TRUS)-guided biopsy, have limitations due to their nonspecific characteristics, their invasive nature, or both [6]. In 2014, Cambridge University established that as many as half of all patients who participated in their study were at risk of PCa misdiagnosis because of inaccurate examination techniques [7]. Therefore, an effective method for the early detection of PCa with high sensitivity and specificity, and noninvasiveness is urgently needed in the clinic.

Magnetic resonance imaging (MRI) shows much better spatial resolution and soft issue contrast than other models of assessment and has been recognized as the best non-invasive method for prostate examination [8-10]. T2-weighted imaging (T2WI) is a conventional MRI method that has been mature for nearly 20 years, but it seems that the sensitivity and specificity are not accurate as we expected. To improve the diagnostic accuracy of T2WI, multi-parametric MRI (mp-MRI) sequences such as dynamic contrast-enhanced (DCE) imaging, diffusionweighted imaging (DWI), and magnetic resonance spectroscopy (MRS) have been recommended as adjuncts to conventional T2WI. These functional MRI techniques can provide metabolic information, display altered cellularity, and aid in the noninvasive characterization of tissue and tumor vascularity [11].

In 1971, Folkman [12] was the first to propose that tumor angiogenesis plays an important role in tumor development, invasion and metastasis across various stages. Other studies later showed that angiogenesis during the pathological staging of PCa could be used as a separate index to assess the formation and development of the disease [13]. DCE-MRI can be used to assess tissue and tumor vascular properties and is a rapidly evolving and noninvasive MRI technique. Although DCE is recommended by the European Society of Urogenital Radiology (ESUR) as a valuable functional technique in PCa detection [6], the accuracy of this method has seldom been systematically reviewed. Therefore, we performed a systematic review and meta-analysis of this technique to examine its diagnostic accuracy in the context of PCa.

\section{RESULTS}

\section{Literature search}

Based on our comprehensive computerized search strategy, Figure 1 provides an overview of the literature search and study selection. Our search yielded 1411 unique records in PubMed,856 unique records in Embase and 95 unique records in Cochrane Library and CENTRAL. Of these, 1748 citations were assessed for eligibility, and the remaining 594 were rejected because they were duplicates. After reviewing the full articles, 441 studies were ultimately excluded for failing to meet the inclusion criteria. Articles were excluded if they did not use DCE-
MRI techniques $(n=18)$; did not provide sufficient available data to construct a $2 \times 2$ table $(n=322)$; were review articles $(n=82)$ or studies focused on recurrent cancer and radiation $(n=19)$. These exclusions yielded a final set of 26 studies for inclusion in the systematic review [14-39]. There were no disagreements between the authors about the number of studies eligible for inclusion.

Of the 26 studies included in our review, two calculated per-regions and per-patients [26, 29]. We identified 21 studies that included DCE combined with other imaging techniques studies of PCa for localization of the tumor (peripheral zone, transition zone and whole gland [14-34], and 7 studies investigated each imaging technique performed per-patients analysis [26, 29, 35-39].

\section{Characteristics of the included studies}

The selected studies included a total of 2070 patients. A total of 1163 patients were included in perregions studies; 1055patients were included in per-patients studies. Patient age ranged from 39-88 years, and the average sample size across studies was 79 participants (range $=16-555)$. Across all studies, the PSA value ranged from $0.18-568.5 \mathrm{ng} / \mathrm{ml}$.

Table 1 summarizes the patient, technical, and study characteristics.

The characteristics of the included studies are illustrated separately in Table 2. Supplementary Table 1 shows individual study results from included studies.

\section{Methodological quality assessment}

The majority of studies were considered to have a high risk of bias for the patient selection $(57 \%, 15 / 26)$, index test $(8 \%, 2 / 26)$. The 4 studies for which the risk of bias for patient selection was unclear did not report exclusion criteria or whether or not the sample was consecutive.

The results of the QUADAS-2 assessment are presented in Figure 2A-2B. All included studies fulfilled the 7 criteria of the QUADAS-2 regarding methodological quality.

\section{Investigations of heterogeneity}

The heterogeneity tests of the sensitivity and specificity values were calculated, respectively. Highly significant heterogeneity was detected when all of the studies were pooled. We chose a random-effect model provided by the software. A single-factor metaregression analysis and the subgroup analysis contributed significantly to this heterogeneity.

\section{Publication bias}

The non-significant slope of Deeks' funnel plot asymmetry test $(P>0.1)$ indicates the absence of publication bias. No significant publication bias was observed for the whole gland $(P=0.91)$ and peripheral zone $(P=0.87)$. 


\begin{tabular}{cccc}
\hline Modality & $\begin{array}{c}\text { No. of Articles } \\
\text { (Whole gland/PZ/TZ) }\end{array}$ & $\begin{array}{c}\text { No. of individuals } \\
\text { (Whole gland/PZ/TZ) }\end{array}$ \\
\hline By biopsy & DCE & $12 / 6 / 2$ & $8781 / 3040 / 563$ \\
DWI & $8 / 5 / 1$ & $7242 / 2285 / 1117$ \\
DCE and DWI & $2 / 1 / 0$ & $2133 / 709 / \mathrm{ND}$ \\
MRS & $2 / 1 / 0$ & $854 / 31 / \mathrm{ND}$ \\
T2 & $11 / 6 / 4$ & $7187 / 3463 / 1558$ \\
DCE and T2 & $2 / 0 / 1$ & $1088 / \mathrm{ND} / 42$ \\
DWI and T2 & $1 / 0 / 2$ & $424 / \mathrm{ND} / 678$ \\
& $7 / 1 / 3$ & $4900 / 1134 / 1246$ \\
& DCE, DWI, and T2 & $1 / 0 / 0$ & $605 / \mathrm{ND} / \mathrm{ND}$ \\
DCE, DWI, MRS and T2 & 2 & 122 \\
DCE & 2 & 120 \\
DWI & 3 & 145 \\
T2 & 2 & 598 \\
& DCE and T2 & 2 & 90 \\
DWI and T2 & 4 & 276 \\
& DCE, DWI, and T2 & 2 & 142 \\
& DCE, DWI, MRS and T2 & 1 & 70 \\
\hline T2 and MRS & & $2 ; 12$
\end{tabular}

Abbreviations: DCE: dynamic contrast-enhanced magnetic resonance; DWI: diffusion weighted imaging; T2: T2 weighted imaging; MRS: magnetic resonance spectroscopy; WR: whole region; PZ: peripheral zone; TZ: transition zone; ND: no data.

\section{Biopsy-level analysis of diagnostic performance summary estimates}

DCE-MRI was compared on the whole gland in 12 studies that provided sufficient information for inclusion in a meta-analysis. The sensitivity varied from 0.25 to 0.96 , and the specificity ranged from 0.67 to 0.96 . The pooled sensitivity was 0.53 (95\% CI 0.39 to 0.67$)$, and the pooled specificity was 0.88 ( $95 \%$ CI 0.83 to 0.92$)$. Overall, DCE had a diagnostic odds ratio (DOR) of 8.69 (95\% CI 4.79 to 15.78 ) and an area under the curve (AUC) of 0.84 ( $95 \%$ CI 0.80 to 0.87 ). Figure $3 \mathrm{~A}$ shows the sensitivity and specificity of the individual studies, pooled estimates and SROC curve.

The diagnostic accuracy of DCE-MRI in the peripheral zone was reported in 6 studies, and the sensitivity estimates and specificity ranged from 0.29 to 0.96 and 0.56 to 0.97 . The pooled $(95 \% \mathrm{CI})$ estimates for sensitivity and specificity were $0.70(95 \%$ CI 0.46 to 0.86 ) and 0.88 (95\% CI 0.76 to 0.94$)$, respectively. DOR was 16.7 (95\% CI 3.77 to 74.72 ), and the AUC was 0.88 ( $95 \%$ CI 0.85 to 0.91 ). Figure 3B shows the sensitivity and specificity of the individual studies, pooled estimates and SROC curve.

Two studies reported the results of transition zone at biopsy of DCE-MRI. In the study by Kim JK et al. [20], which enrolled 53 participants, the sensitivity was 0.96 (95\% CI 0.91 to 0.98$)$, and the specificity was 0.51 (95\%
CI 0.44 to 0.59$)$.In the study by Reisaeter et al. [23], which enrolled 63 participants, the sensitivity was 0.13 (95\% CI 0.05 to 0.25 ), and the specificity was 0.97 (95\% CI 0.95 to 0.98 ). Figure $3 \mathrm{C}$ shows the sensitivity and specificity of the individual studies.

\section{Studies directly comparing tests}

\section{Comparative analysis: DCE versus DWI}

For the whole gland, eight studies involving 544 patients reported DCE compared with DWI and provided sufficient information for inclusion in a meta-analysis. DWI appeared to have a statistically higher pooled sensitivity $(P<0.001)$; the pooled estimates for sensitivity and specificity were 0.43 (95\% CI 0.36 to 0.51$)$ and 0.90 ( $95 \%$ CI 0.84 to 0.94 ) for DCE and 0.54 (95\% CI 0.42 to $0.67)$ and 0.89 (95\% CI 0.83 to 0.92$)$ for DWI.

When we included four studies of the peripheral zone involving 325 patients that reported DCE compared with DWI, DWI appeared to have a statistically higher pooled sensitivity $(P=0.0498)$. The pooled estimates for sensitivity and specificity were 0.56 (95\% CI 0.35 to 0.74 ) and 0.82 (95\% CI 0.69 to 0.91$)$ for DCE and 0.67 (95\% CI 0.45 to 0.84 ) and 0.81 (95\% CI 0.57 to 0.93 ) for DWI.

For the transition zone, Reisaeter et al. [23] in a study of 63 patients reported DCE compared with DWI. DCE and DWI appeared to have similar sensitivity, 
respectively, with values of 0.13 (95\% CI 0.05 to 0.25 ) versus 0.17 (95\% CI 0.08 to 0.29 ), and specificity, with values of 0.97 (95\% CI 0.95 to 0.98$)$ versus 0.94 (95\% CI 0.91 to 0.96$)$, respectively.

Figure $4 \mathrm{~A}-4 \mathrm{C}$ shows the sensitivity and specificity of the individual studies, pooled estimates and SROC plot with the $95 \%$ confidence region.

\section{Comparative analysis: DCE versus T2}

For the whole gland, ten studies involving 483 patients reported DCE compared with T2 and provided sufficient information for inclusion in a meta-analysis. There was evidence that DCE had better test accuracy than T2.
Using the results from the earlier analysis, DCE appeared to have higher pooled sensitivity $(P=0.1666)$ and statistically higher specificity $(P<0.001)$. The pooled estimates for sensitivity and specificity were 0.52 (95\% CI 0.36 to 0.68 ) and $0.89(95 \%$ CI 0.82 to 0.93$)$ for DCE and $0.50(95 \% \mathrm{CI}$ 0.41 to 0.58 ) and 0.85 (95\% CI 0.75 to 0.91$)$ for T2.

For the peripheral zone, five studies involving 220 patients reported DCE compared with T2. The pooled estimates for sensitivity and specificity were 0.71 (95\% CI 0.42 to 0.89 ) and $0.89(95 \%$ CI 0.75 to 0.95$)$ for DCE and 0.59 (95\% CI 0.46 to 0.72 ) and $0.73(95 \%$ CI 0.57 to 0.84$)$ for T2. These results indicate that DCE had statistically higher sensitivity $(P=0.0194)$ and specificity $(P=0.006)$ in the peripheral zone compared with $\mathrm{T} 2$.

PRISMA 2009 Flow Diagram

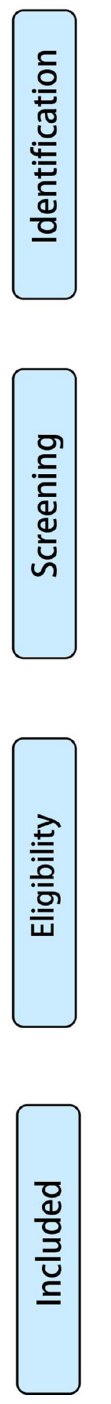

Records identified from Pubmed searching $(n=1411)$

Records identified from Embase searching $(n=856)$

Records identified from Cochrane Library and CENTRAL searching $(n=95)$

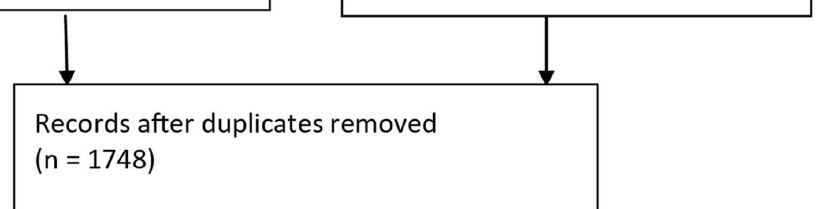

1281 records excluded after screening of titles and abstracts A.Letters, editorials B.Protocols C.Animal studies D.Duplicate publications

441 articles excluded with reasons A.18 studies did not use DCE MRI techniques B.322 studies cannot calculate $2 \times 2$ contingency tables C.82 studies were review articles D.19 studies focused on recurrent and radiation.

Studies included in quantitative synthesis $(\mathrm{n}=26)$

Figure 1: PRISMA 2009 flow diagram. 
Table 2: The characteristics of all the studies included

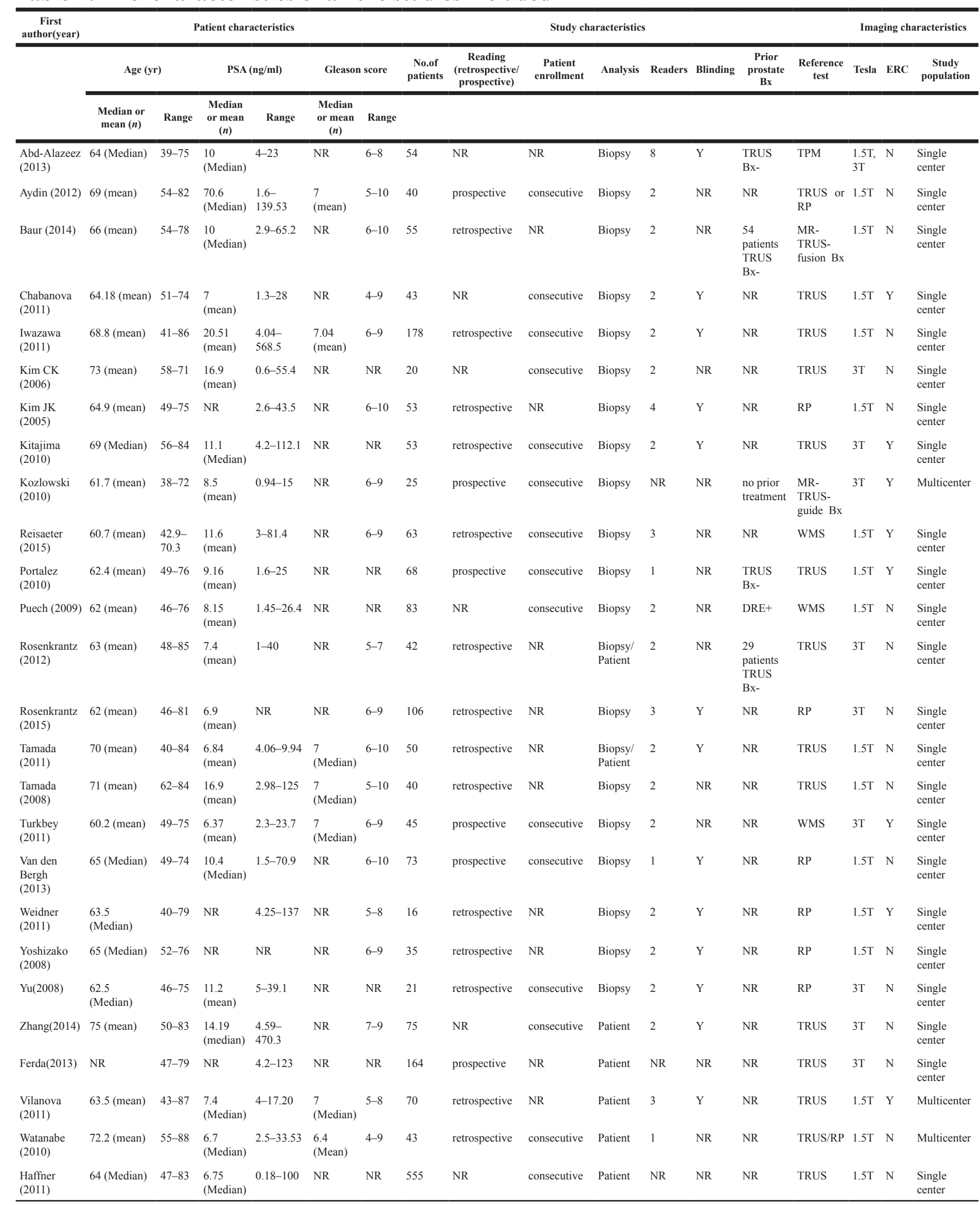

Abbreviations: NR: not reported; ERC: endorectal coil; TRUS: transrectal ultrasound; Bx: biopsy; Bx-: negative TRUS-guided Bx; TPM: transperineal template prostate mapping; WMS: whole-mount section histopathology; DRE+: positive digital rectal examination; RP: radical prostatectomy. 
For the transition zone, two studies involving 116 patients reported DCE compared with T2.In Reisaeter et al. [23], the sensitivity was 0.13 ( $95 \%$ CI 0.05 to 0.25 ), and the specificity was 0.97 (95\% CI 0.95 to 0.98$)$ for DCE, whereas the sensitivity was 0.13 (95\% CI 0.05 to 0.25$)$, and the specificity was 0.94 (95\% CI 0.92 to 0.96$)$ for T2. In Kim JK et al. [20], the sensitivity was 0.96 (95\% CI 0.91 to 0.98 ), and the specificity was 0.51 ( $95 \%$ CI 0.44 to 0.59 ) for DCE, whereas the sensitivity was 0.45 ( $95 \%$ CI 0.37 to 0.98$)$, and the specificity was $0.73(95 \%$ CI 0.66 to 0.79$)$ for $\mathrm{T} 2$.

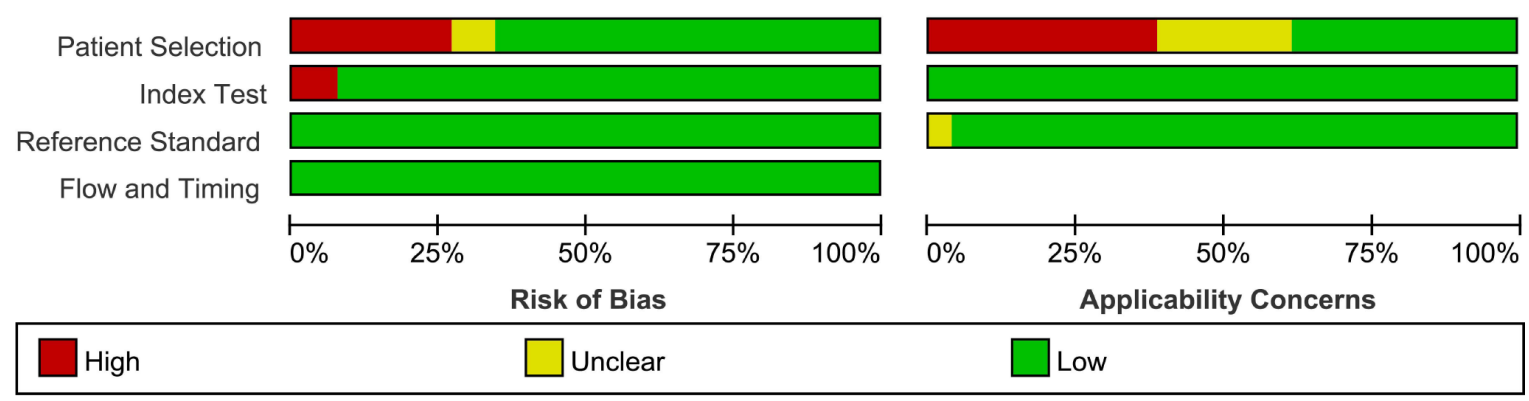

A

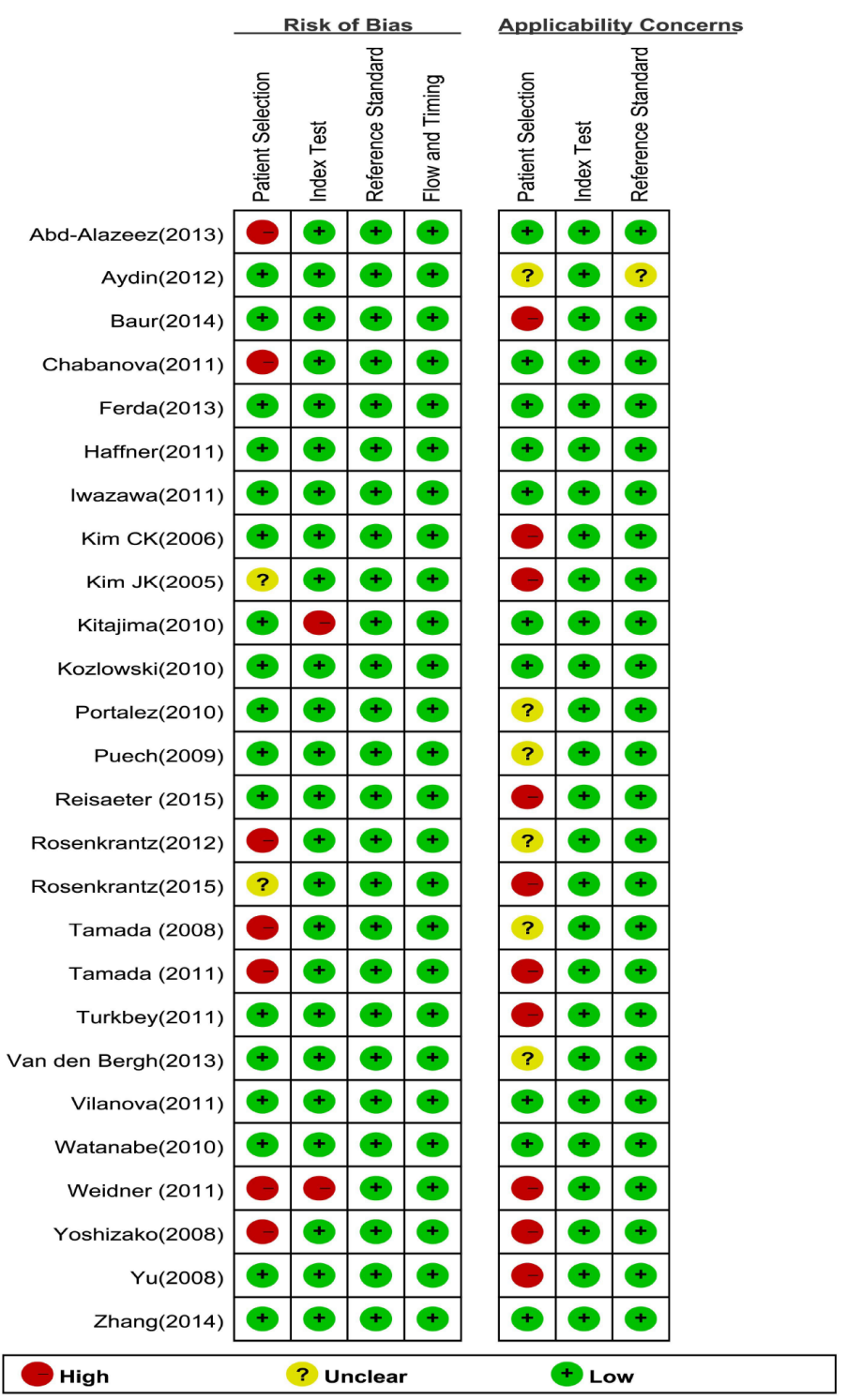

B

Figure 2: Methodological quality graph and summary. 
Figure 5A-5C shows the sensitivity and specificity of the individual studies, pooled estimates and SROC plot with the $95 \%$ confidence region.

\section{Comparative analysis: DCE versusT2 combined with DCE and DWI}

For the whole gland, five studies involving 281 patients reported DCE compared with DCE versus T2 combined with DCE and DWI and provided sufficient information for inclusion in a meta-analysis. The pooled estimates for sensitivity and specificity were $0.41(95 \% \mathrm{CI}$ 0.33 to 0.52$)$ and $0.92(95 \% \mathrm{CI} 0.89$ to 0.95 ) for $\mathrm{DCE}$, and 0.61 (95\% CI 0.53 to 0.68 ) and 0.90 (95\% CI 0.85 to 0.93 ) for T2 combined with DCE and DWI, it shows statistically difference in sensitivity $(p<0.0001)$.

For the peripheral zone, Reisaeter et al. [23] reported in a study involving 63 patients.T2 combined with DCE and DWI appeared to have slightly higher sensitivity 0.60 ( $95 \%$ CI 0.55 to 0.66 ) versus DCE 0.51 (95\% CI 0.45 to 0.57 ) and similar specificity.

For the transition zone, Reisaeter et al. [23] reported DCE compared with T2 combined with DCE and DWI. The sensitivity and specificity were 0.13 (95\% CI 0.05 to 0.25 ) and 0.97 (95\% CI 0.95 to 0.98 ) for DCE and 0.15 ( $95 \%$ CI 0.07 to 0.27 ) and 0.95 (95\% CI 0.93 to 0.97 ) for T2 combined with DCE and DWI. Thus, T2 combined with DCE and DWI appeared to have slightly higher sensitivity.

Figure $6 \mathrm{~A}-6 \mathrm{C}$ shows the sensitivity and specificity of the individual studies, pooled estimates and SROC plot with the $95 \%$ confidence region.

\section{Other direct comparison tests}

To assess other direct comparison tests, data were derived from only one or two studies. Because of the clear evidence of incorporation bias, we did not perform a metaanalysis of measures of test accuracy.
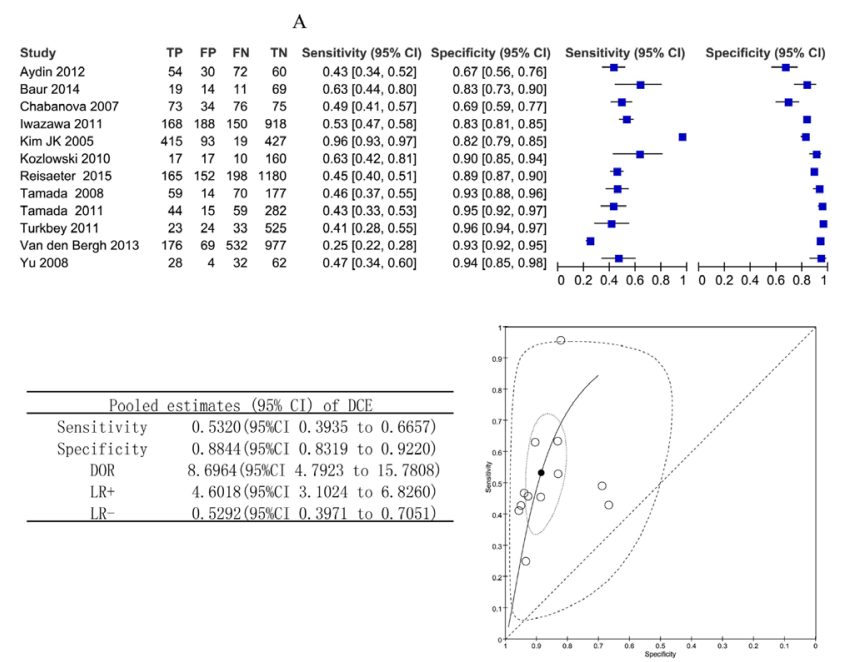

Aydin [15], Turkbey [30] and Weidner [32] directly compared DCE and MRS. For the whole gland, Aydin [15] reported DCE slightly higher specificity and lower sensitivity compared to MRS, whereas Turkbey [30] reported the slightly lower specificity and higher sensitivity compared to MRS. For the peripheral zone, MRS had slightly higher specificity and similar sensitivity. Iwazawa [18] showed that DCE combined with DWI had significantly higher sensitivity than DCE.

Supplementary Table 2 shows the results of the other direct comparison as above.

\section{Studies indirectly comparing tests for the biopsy- level pooled estimates}

For the biopsy-level estimates of tests with four or more studies, compared with DWI, DCE was observed to have equivalent sensitivity and specificity on whole gland, and have higher sensitivity, specificity and diagnosis accuracy than T2.

Supplementary Table 3 shows the results for the biopsy-level pooled estimates from indirect comparisons.

\section{Studies for patient-level analysis}

There was none of test included in four or more studies. So we carried out no meta-analyses due to insufficient data for pooling on any outcome. therefore, we reported outcomes narratively and summarised in tabular format.

Supplementary Table 4 shows the results for the patient-level analysis.

\section{Subgroup analysis}

Significant heterogeneity were observed, As we used subgroup and meta-regression analysis that may conducted with regard to different study characteristics. The source of heterogeneity mainly revealed the method of DCE

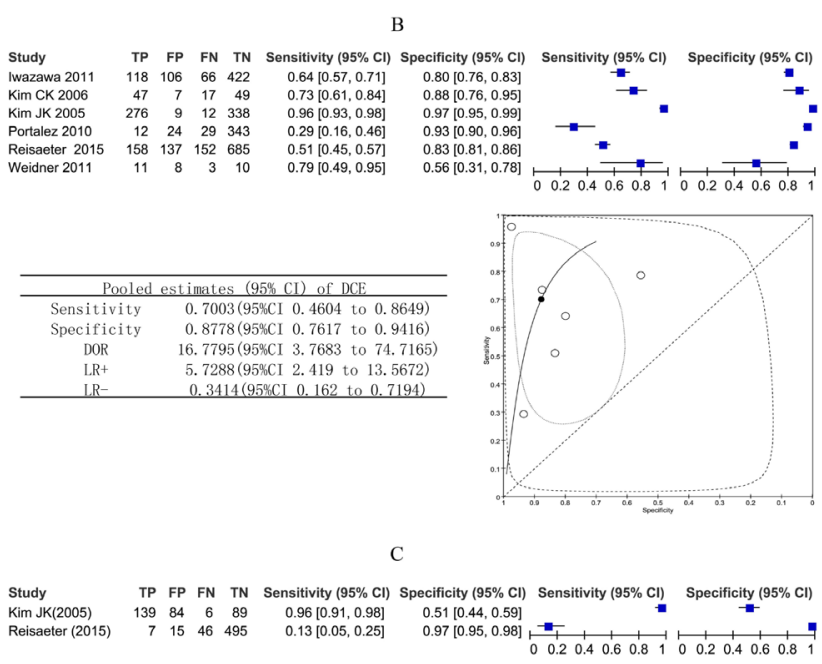

Figure 3: (A-C) Biopsy-level analysis of DCE. Dynamic contrast-enhanced MRI biopsy-level analysis: forest plots, pooled estimates and SROC curve showed in whole gland (A) and peripheral zone (B), forest plot showed in transition zone (C). 
technology ( $p=0.0093)$. but we should not neglected that the heterogeneity due to the heterogeneous nature of the related studies and inevitable in clinical practice. The subgroup analyses showed the studies by using quantitative methods and 3.0 Tesla had higher sensitivity and specificity.

Table 3 contains results of this subgroup analysis.

\section{DISCUSSION}

There are different opinions regarding the added value of DCE sequences. For example, according to the new PI-RADS v2 guideline [40], DCE-MRI technology is an essential component of the mp-MRI prostate examination. However, DCE is not recommended as a mpMRI approach for PCa detection by the National Institute for Health and Care Excellence (NICE) guidelines [41].

In this study, it was showed that the specificity values for detection $\mathrm{PCa}$ on both whole gland and $\mathrm{PZ}$ were approximately 0.88 , indicating that DCE-MRI can distinguish between normal tissues from PCa. Biger et al. demonstrated that PCa was associated with an approximately two-fold increase in the total number of vessels seen on histologic sections [42]. Based on the findings from Biger et al., Engelbrecht et al. reported that PCa showed more pronounced enhancement resulting in higher signal on DCE-MRI than surrounding normal prostate tissues [43]. Therefore, DCE-MRI shows its ability in the localizing of PCa. Moreover, it has high accuracy in detecting cancer recurrence who have undergone radical prostatectomy [44] or post-radiotherapy patients [45]. Meanwhile, our direct comparison study showed that the specificity of DCE was statistically superior to T2WI ( 0.89 vs $0.73, p<0.0001)$. The study by Shimizu et al. also showed that low specificity $(0.70)$ and sensitivity (0.29) limited the use of T2WI [46]. T2WI was a standard sequence in detecting $\mathrm{PCa}$, in which tumor tissue appears hypointense relative to the normal
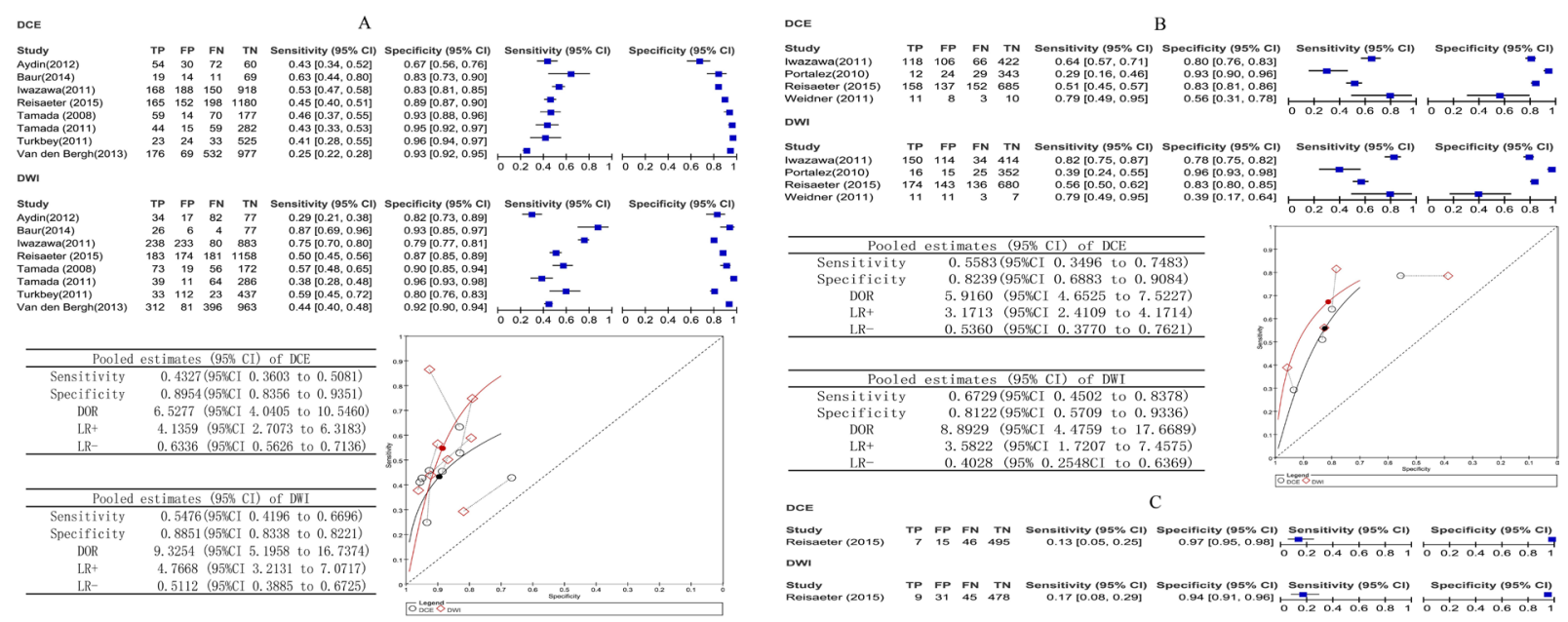

Figure 4: (A-C) Comparative analysis DCE versus DWI. Dynamic contrast-enhanced MRI compared with DWI biopsy-level analysis: forest plots, pooled estimates and SROC curve showed in whole gland (A) and peripheral zone (B), forest plot showed in transition zone (C).
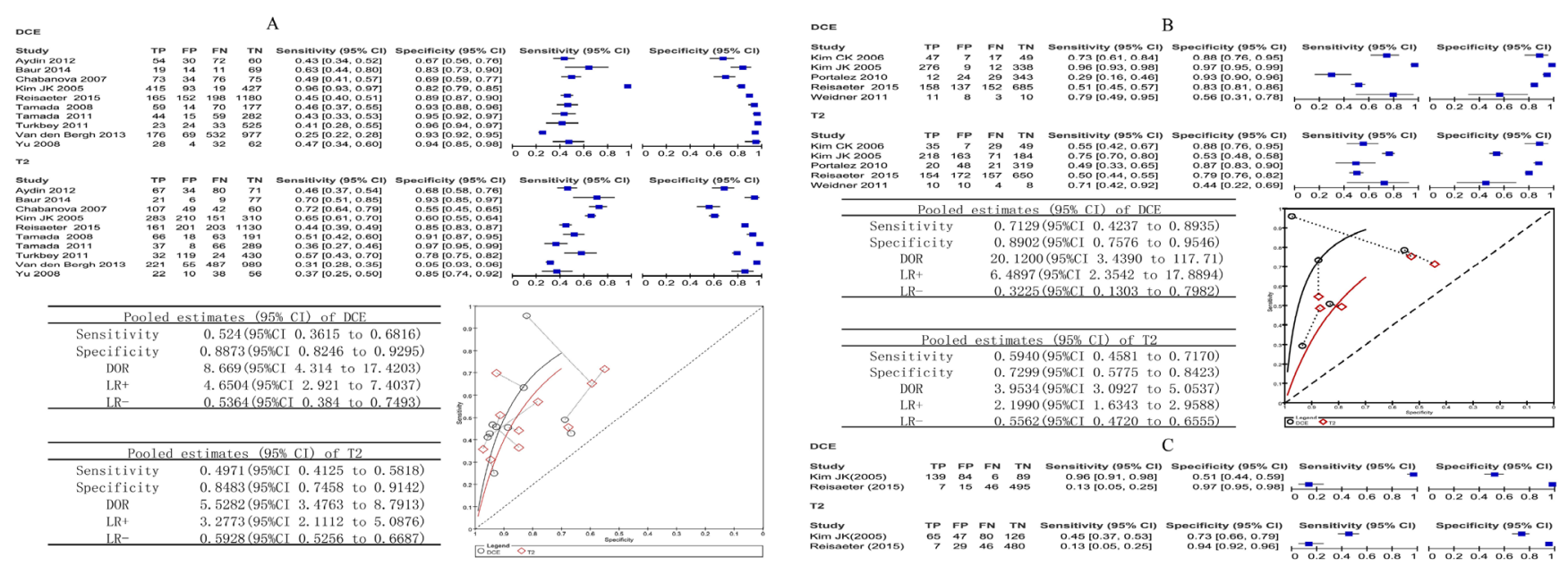

Figure 5: (A-C) Comparative analysis DCE versus T2. Dynamic contrast-enhanced MRI compared with T2 biopsy-level analysis: forest plots, pooled estimates and SROC curve showed in whole gland (A) and peripheral zone (B), forest plot showed in transition zone (C). 
Table 3: Subgroup analysis and meta-analysis

\begin{tabular}{|c|c|c|c|c|c|c|c|}
\hline Study characteristics & No & sensitivity & specificity & DOR & $\begin{array}{c}\text { Chi2 test of } \\
\text { heterogeneity }\end{array}$ & $\begin{array}{c}P \text { value for } \\
\text { heterogeneity }\end{array}$ & $P$-value \\
\hline Total & 12 & $0.53(0.39-0.67)$ & $0.88(0.83-0.92)$ & $8.70(4.80 .-15.78)$ & 181.08 & & \\
\hline Field strength & & & & & & & 0.2326 \\
\hline $1.5 \mathrm{~T}$ & 9 & $0.54(0.39-0.70)$ & $0.86(0.81-0.91)$ & $7.34(3.81-14.15)$ & 170.72 & $<0.001$ & \\
\hline $3.0 \mathrm{~T}$ & 3 & $0.50(0.22-0.79)$ & $0.94(0.89-0.99)$ & $15.9(10.27-24.62)$ & 0.05 & 0.947 & \\
\hline Coil & & & & & & & 0.796 \\
\hline Without endorectal coil & 8 & $0.55(0.38-0.72)$ & $0.88(0.83-0.94)$ & $10.11(4.68-21.85)$ & 153.44 & $<0.001$ & \\
\hline With endorectal coil & 4 & $0.49(0.25-0.73)$ & $0.89(0.81-0.96)$ & $7.22(3.18-16.37)$ & 28.02 & $<0.001$ & \\
\hline Blind & & & & & & & 0.5910 \\
\hline Unblind & 5 & $0.51(0.29-0.73)$ & $0.87(0.80-0.95)$ & $8(3.87-16.55)$ & 35.20 & $<0.001$ & \\
\hline Blind & 7 & $0.55(0.37-0.73)$ & $0.89(0.84-0.94)$ & $10.10(4.24-24.03)$ & 145.55 & $<0.001$ & \\
\hline Patient enrollment & & & & & & & 0.1051 \\
\hline Non-consecutive or unclear & 4 & $0.69(0.50-0.88)$ & $0.90(0.83-0.96)$ & $19.37(5.49-68.36)$ & 43.68 & $<0.001$ & \\
\hline consecutive & 8 & $0.45(0.30-0.60)$ & $0.88(0.82-0.93)$ & $6.12(3.98-9.39)$ & 52.13 & $<0.001$ & \\
\hline Methods & & & & & & & 0.0093 \\
\hline Traditional methods & 5 & $0.36(0.33-0.39)$ & $0.89(0.88-0.90)$ & $8.62(5.6-12.26)$ & 14.71 & 0.005 & \\
\hline Semi-parametric methods & 3 & $0.48(0.42-0.54)$ & $0.72(0.67-0.77)$ & $2.75(1.20-6.57)$ & 9.77 & 0.008 & \\
\hline parametric methods & 4 & $0.68(0.49-0.88)$ & $0.90(0.84-0.96)$ & $19.92(4.63-85.61)$ & 91.99 & $<0.001$ & \\
\hline
\end{tabular}

Abbreviations: DOR: diagnostic odds ratio.

peripheral tissue [47]. However, focal decreases in T2 signal intensity can result from benign processes and $\mathrm{PCa}$ may also show minimally reduced T2 signal, making them nearly isointense on T2WI [48]. The reports from Rosenkrantz et al. suggested that DCE sequences were mainly effective in detecting non-nodular, infiltrating vascular lesions, which were poorly visible on T2WI or DWI [49].

However, the sensitivity value of DCE-MRI for locating PCa was relatively low on the whole gland $(0.55)$ and PZ (0.70), suggesting that DCE-MRI had difficulty in distinguishing between malignant tumors and benign lesions. Agha et al. reported that some benign prostatic lesions, such as benign prostatic hyperplasia (BPH), may show enhancement pattern of nearby criteria to the $\mathrm{PCa}$ enhancement curves, and some unavoidable technical errors, such as rectal motions, may distort the relatively long timed dynamic sequences [50]. Although Tofts et al. [51] standardized acquisition parameters of quantitative analysis, there is also a problem of the rate constants overlapping between benign and malignant tissues [52]. Meanwhile, our direct comparison reflected that the sensitivity values of DCE were statistically lower on whole gland and relatively lower on PZ than the values from DWI. This could be explained at least in part by different approaches to evaluate DCE-MRI in a qualitative

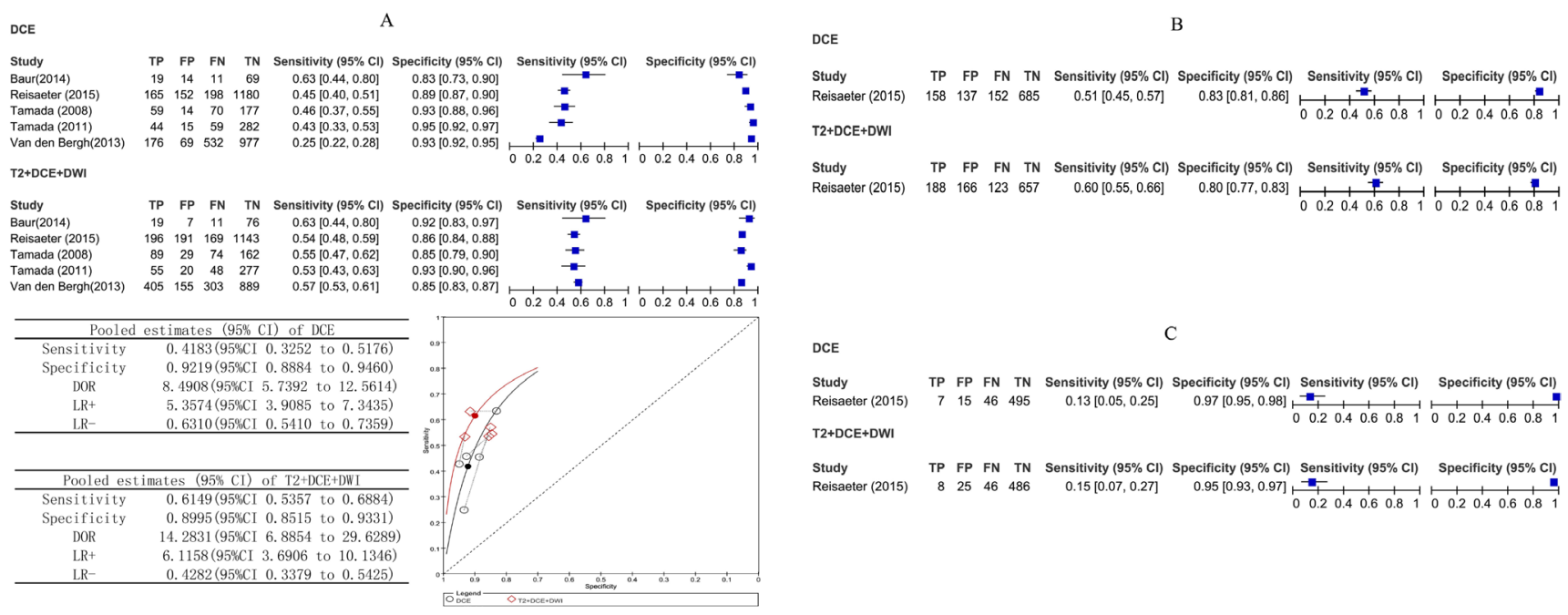

Figure 6: (A-C) Comparative analysis DCE versus T2 combine DCE and DWI. Dynamic contrast-enhanced MRI compared with T2 combine DCE and DWI biopsy-level analysis: forest plot, pooled estimates and SROC curve showed in whole gland (A), forest plots showed in peripheral zone (B) and in transition zone (C). 
or quantitative way [16]. However, Iwazawa et al. suggested that the prostate lesions were also missed when detection was attempted by DWI alone. Thus, excluding DCE from routine prostate MRI incurs a risk of failure in the detection of PCa, especially for lesions in the PZ [18].

By our subgroup and meta-regression analysis, it was found that the main source of heterogeneity was from the method of DCE technology. Compared to the qualitative and the semi-parametric method, parametric methods have a slight improvement in the sensitivity and DOR. Qualitative DCE-MRI is based on the subjective evaluation by the experience of the observer and semiparametric uses signal intensity time curves (SI) to classify the tissue. Both qualitative and semi-parametric methods directly or indirectly focus on evaluating the strength of enhancement of the regions of interest (ROIs) [53]. However, based more on simulating the change in the concentration agent using eligible pharmacokinetic (PK) modeling techniques, the parametric method can determine the rate of contrast exchange and derived quantitative modeling parameters such as Ktrans and Kep [54]. This method, consistent with the theory by Folkman [12], not only further computerizes empirical parameters, but also reduces the bias among different observers.

Finally, we acknowledge certain limitations of this meta-analysis. Firstly, TZ tumors are estimated to account for approximately $30 \%$ of PCa cases and pose a clinical challenge because of the difficulty of their detection [55] despite compelling data supporting the value of MRI for improving PZ tumor detection [56].The actual clinical utility of MRI for improving TZ tumor detection was still unclear. Sinnott et al. inferred that different prostate zones could point a key source of variability in PCa prognosis and treatment response [57]. Therefore, PZ, TZ, and whole gland should not be combined in a single test due to the different incidence of the disease, which may have an impact on the diagnostic performance. Villers et al. found that DCE-MRI was an accurate technique for detecting and quantifying TZ tumor with early enhancement which had no suspicious on T2WI [58]. However, Hoeks et al. reported that DWI and DCE may not improve TZ cancer detection and localization accuracy compared with T2WI [59]. Our study had included 4 literatures [20, 23, 27, 33], which had focused on TZ, but it was insufficient for a meaningful pooling estimate results. Therefore, the effects on TZ tumor detection need to be clarified with more literatures in the near future.

Secondly, according to the method by Cheikh et al., who found that "per-region" and "per-patient" analysis may have a dramatic difference in localizing PCa [60], we prospectively designed the 'per-patient' analysis. The sensitivity and specificity showed dramatic differences compared to 'per-region' analysis across the included studies. Our results were consistent with Cheikh et al. , who suggested that 'per-region' analysis could lead to an overestimation of specificity due to high proportion of regions without cancer. In our analysis, there was insufficient data for pooling results and the meaningful pooling results need more research support.

Lastly, most studies used a combination of targeted and systematic biopsies. Although biopsies have been recommended as an gold standard in detected $\mathrm{PCa}$ in several guidelines [61-62], it might tend to miss tumors on first systematic biopsy [63], and the diagnostic accuracy might therefore be slightly interfered. Some of the individual studies had limited quality, which may influenced our meta-analysis outcomes.

\section{MATERIALS AND METHODS}

\section{Study registration}

The protocol was registered at the international prospective register of systematic reviews (PROSPERO) website under number CRD42017056236 [64]. This study followed the PRISMA statement and the Cochrane Handbook for Diagnostic Test Accuracy Reviews [65-66].

Institutional review board ethical approval was not needed because of the reviewing nature of this study.

\section{Criteria for considering studies for this review}

\section{Type of studies}

We first included a study with sufficient data to evaluate the diagnostic accuracy of DCE-MRI for the diagnosis of PCa using histopathological assessment as the reference standard.

We also performed direct and indirect comparisons with other MRI methods and to explore the strengths and shortcomings of DCE-MRI [67].

\section{Participants}

We included adult patients with clinical symptoms suspicious for PCa who underwent DCE-MRI examination and biopsy.

\section{Index test}

Studies that assessed the accuracy of diagnostic tests included DCE with or without other methods of imaging such as MRS, T2WI or DWI alone or in combination were included.

\section{Reference standards}

The reference standard was histopathological assessment of biopsied tissue. Tissue samples were obtained by surgery, autopsy, or TRUS-guided biopsy. The units of analyses reported by the studies included biopsy, site, segment, region and core. 


\section{Types of outcomes}

The primary objective of our study is to evaluate the diagnostic capability of DCE-MRI in patients suspected of having PCa. A secondary outcome is to compare DCE with other MRI methods such as DWI, T2WI, MRS to explore the strengths and shortcomings of DCE.

\section{Literature search strategy}

Two reviewers performed a comprehensive literature search to identify relevant studies published in English. An electronic search of articles published from January 2000 to September 2016 was performed using PubMed and Embase by incorporating the following keywords: "prostatic OR prostate tumor OR prostate cancer OR prostate carcinoma" AND "magnetic resonance imaging OR MRI OR magnetic resonance" AND "contrast OR contrast medium OR DCE ORDCE-MRI OR dynamic OR contrast enhance". Other sources such as the Cochrane Library were also checked for relevant studies using similar keywords. Abstracts were reviewed for relevance to the defined review question. If it was not clear from the abstract whether the paper might contain relevant data, the full paper was assessed.

Supplementary Table 5 shows the search strategies for the searches.

\section{Study selection and data extraction}

Two reviewers independently conducted the study selection protocol. Articles that were not excluded by at least one investigator were scrutinized by checking the full text independently. Final inclusion was determined after discussion to resolve any discrepancies. Duplicate use of the same data was carefully excluded.

A study was included for review if it met the following inclusion criteria: A. the research focused on the preoperative diagnosis of prostate cancer; B. DCEMRI was performed with a gadolinium agent and used to identify and characterize prostate cancer; $\mathrm{C}$. the study incorporated the 'gold standard' of histopathological analysis performed during surgery or autopsy, or a TRUSguided biopsy was used as the reference standard; and D. sufficient data were presented directly or indirectly to calculate $2 \times 2$ contingency tables for per-patient or perlesion statistics.

The following exclusion criteria were also applied: A. all review articles, letters, comments, case reports and non-clinical trials were eliminated from consideration; B. studies with fewer than 10 patients in the sample were excluded; C. Studies featuring patients with previous recurrences and who have received radiation therapy for prostate cancer will be excluded.

To conduct a reliable analysis, the following data were extracted: patient age, number of previous prostate biopsies, magnetic field strength (in Tesla), use of an endorectal coil, use of other coils, DCE-MRI acquisition parameters, use of additional techniques, year of publication, study population, reference standard (i.e. prostate biopsy or prostatectomy specimen), patient enrollment, study design, blinding, number of readers.

\section{Quality assessment}

The same two review authors independently assessed the relevant extracted data. Any disagreements between the two reviewers were resolved by a third reviewer. The two reviewers each used 7 items from the published quality assessment of diagnostic accuracy studies version 2 (QUADAS-2) guidelines to assess the relevant studies. The QUADAS-2 tool is structured in a series of questions that should be answered 'yes', 'no', or 'unclear' and aims to evaluate study quality involving the participant spectrum, index test, reference standard, and flow and timing as 'high risk' 'low risk' and 'unclear'. As a general rule, if a particular point was not mentioned in a document, then the relevant item of signaling was marked as 'unclear'. If at least one answer to the signaling questions was 'no' or at least two answer 'unclear' of the four domains and applicability, we classified the studies as high risk of bias [68].

We summarized the methodological quality using the risk of bias and applicability concerns. Supplementary Table 6 shows the adopted items that served the purposes of our review.

\section{Data synthesis and analysis}

The numbers of true positive (TP), false positive (FP), false negative (FN) and true negative (TN) findings with regard to MRI used to diagnose preoperative PCa were calculated from the included studies to construct $2 \times 2$ Tables.

We used data from the $2 \times 2$ tables to calculate sensitivity, specificity, positive likelihood ratios (LR+), and negative likelihood ratios (LR-) with 95\% CIs and accuracy for each study. A continuity correction of 0.5 was added to all cells for studies that contained a count of zero to avoid potential problems with odds calculations for studies with sensitivity or specificity values of $100 \%$.

The diagnostic odds ratio (DOR) was estimated based on the LR+ and LR - to represent the odds of a positive test among patients with PCa compared with the odds of a positive image among patients without $\mathrm{PCa}$. This single indicator of test accuracy included a combination of sensitivity and specificity information [69]. We also plotted the sensitivity and specificity values to construct a summary receiver operating characteristic (SROC) curve. Then, we calculated the area under the SROC curve (AUC).

The directly comparative analysis was performed between all tests with four or more studies with relevant data. we compared the sensitivity and specificity of the direct comparison method to whether the t-statistics in the output provide statistically significant [70]. The 
comparative analysis consisted of an indirect comparison in which all tests with relevant data were compared and accuracy assuming a common underlying shape.

\section{Investigations of heterogeneity}

Heterogeneity in meta-analysis refers to the degree of variability in study results. If heterogeneity existed, a random effect model was used for the primary metaanalysis to obtain a summary estimate for sensitivity with 95\% CIs. An exploration of the reasons for heterogeneity rather than computation of a single summary measure is an important goal of meta-analysis.

\section{Publication bias}

Publication bias was studied using Deeks funnel plots. The funnel plots was assessed visually by using a scatter plot of the inverse of the square root of the effective sample size (ESS) - $1 / \mathrm{ESS}^{1 / 2}$ — versus the diagnostic log odds ratio (lnDOR), Formal testing for publication bias was conducted by using a regression of $1 \mathrm{nDOR}$ against $1 / \mathrm{ESS}^{1 / 2}$ and weighting according to the ESS, with $P<$ 0.10 indicating significant asymmetry [71].

\section{Subgroup analysis}

If heterogeneity is detected, We using subgroup analysis and meta-regression to investigate the source of heterogeneity. subgroup analyses were performed by the study characteristics such as magnetic field strength, coils, reference standards, patient enrollment, study design, and blinding, the method of DCE technology. Meta-regression analysis was also performed to identify potential factors that could explain the source of heterogeneity [72]

\section{Software}

The tables were entered into RevMan5 software (The Cochrane Collaboration, The Nordic Cochrane Centre, Copenhagen, Denmark) and SAS version 9.3 (SAS Institute Inc., Cary, NC, USA). All statistical analyses and graphical plots were performed in RevMan5.

\section{CONCLUSIONS}

Our meta-analysis demonstrates that although DCEMRI can provide informative supplementary diagnostic accuracy to detect PCa, it remains a confirmatory tool. As new quantitative techniques are developed to enhance the standards of optimal scans, DCE-MRI may attain important clinical status.

\section{Author contributions}

G.J. and Y.Z. contributed for study search, quality check, data extraction. Z.C. work for study search, quality check, data extraction and analysis as a principal investigator. X.L. provided study conception this study. P.L., L.C. and Y.G. and worked for interpretation of the data as radiologists. J.Y. contributed as a meta-analysis specialist and drafting.

J.Y managed the study. All authors reviewed and finally approved the manuscript.

\section{ACKNOWLEDGMENTS}

We thank Wiley for the linguistic assistance during the preparation of this manuscript. This work was supported by Ningxia Natural Science Foundation of China (NZ1234 and NZ13280). National Natural Science Foundation of China (No.81171317, 81471631), the 2011 New Century Excellent Talent Support Plan of the Ministry of Education, China (NCET-11-0438), and National Key Research and Development Plan of China (2016YFC0100300).

\section{CONFLICTS OF INTEREST}

Competing financial interests: The authors declare no competing financial interests.

\section{REFERENCES}

1. Levi F, Lucchini F, Negri E, Boyle P, La Vecchia C. Leveling of prostate cancer mortality in Western Europe. Prostate. 2004; 60:46-52.

2. Siegel RL, Miller KD, Jemal A. Cancer statistics. 2015. CA Cancer J Clin. 2015; 65:5-29.

3. Randazzo M, Beatrice J, Huber A, Grobholz R, Manka L, Chun FK, Recker F, Kwiatkowski M. A "PSA pyramid" for men with initial prostate-specific antigen $\leq 3 \mathrm{ng} / \mathrm{ml}$ : a plea for individualized prostate cancer screening. Eur Urol. 2015; 68:591-597.

4. Garnick MB. Prostate cancer: screening, diagnosis, and management. Ann Intern Med. 1993; 118:804-18.

5. Onyiaorah IV, Ukah CO, Anyiam DC, Onwukamuche ME, Efobi CC. Accuracy of clinical diagnosis of prostate cancer: Implications for patients' management. Trop J Med Res 2015; 18:61-67.

6. Barentsz JO, Richenberg J, Clements R, Choyke P, Verma S, Villeirs G, Rouviere O, Logager V, Fütterer JJ, European Society of Urogenital Radiology. ESUR prostate MR guidelines 2012. Eur Radiol. 2012; 22:746-757.

7. Shaw GL, Thomas BC, Dawson SN, Srivastava G, Vowler SL, Gnanapragasam VJ, Shah NC, Warren AY, Neal DE. Identification of pathologically insignificant prostate cancer is not accurate in unscreened men. Br J Cancer. 2014; 110:2405-2411.

8. Fütterer JJ, Briganti A, De Visschere P, Emberton M, Giannarini G, Kirkham A, Taneja SS, Thoeny H, Villeirs 
G, Villers A. Can clinically significant prostate cancer be detected with multiparametric magnetic resonance imaging? A Systematic Review of the Literature. Eur Urol. 2015; 68:1045-1053.

9. Nishida S, Kinoshita H, Mishima T, Kurokawa H, Sakaida N, Matsuda T. Prostate cancer detection by prebiopsy 3.0-Tesla magnetic resonance imaging. Int J Urol. 2011; 18:653-658.

10. Turkbey B, Choyke PL. Multiparametric MRI and prostate cancer diagnosis and risk stratification. Curr Opin Urol. 2012; 22:310-315.

11. Pinto F, Totaro A, Palermo G, Calarco A, Sacco E, D'Addessi A, Racioppi M, Valentini A, Gui B, Bassi P. Imaging in prostate cancer staging: present role and future perspectives. Urol Int. 2012; 88:125-136.

12. Folkman J. Tumor angiogenesis: therapeutic implications. N Engl J Med. 1971; 285:1182-1186.

13. Brawer MK, Deering RE, Brown M, Preston SD, Bigler SA. Predictors of pathologic stage in prostatic carcinoma: The role of neovascularity. Cancer. 1994; 73:678-687.

14. Abd-Alazeez M, Ahmed HU, Arya M, Charman SC, Anastasiadis E, Freeman A, Emberton M, Kirkham A. The accuracy of multiparametric MRI in men with negative biopsy and elevated PSA level: can it rule out clinically significant prostate cancer? Urol Oncol. 2014; 32:45.e17-22.

15. Aydin H, Kizilgöz V, Tatar IG, Damar C, Ugan AR, Paker I, Hekimoğlu B. Detection of prostate cancer with magnetic resonance imaging: optimization of T1-weighted, T2weighted, dynamic-enhanced T1-weighted, diffusion-weighted imaging apparent diffusion coefficient mapping sequences and MR spectroscopy, correlated with biopsy and histopathological findings. J Comput Assist Tomogr. 2012; 36:30-45.

16. Baur AD, Maxeiner A, Franiel T, Kilic E, Huppertz A, Schwenke C, Hamm B, Durmus T. Evaluation of the prostate imaging reporting and data system for the detection of prostate cancer by the results of targeted biopsy of the prostate. Invest Radiol. 2014; 49:411-420.

17. Chabanova E, Balslev I, Logager V, Hansen A, Jakobsen H, Kromann-Andersen B, Norgaard N, Horn T, Thomsen HS. Prostate cancer: $1.5 \mathrm{~T}$ endo-coil dynamic contrast-enhanced MRI and MR spectroscopy: correlation with prostate biopsy and prostatectomy histopathological data. Eur J Radiol. 2011; 80:292-296.

18. Iwazawa J, Mitani T, Sassa S, Ohue S. Prostate cancer detection with MRI: is dynamic contrast-enhanced imaging necessary in addition to diffusion-weighted imaging? Diagn Interv Radiol. 2011; 17:243-248.

19. Kim CK, Park BK, Kim B. Localization of prostate cancer using 3T MRI: comparison of T2-weighted and dynamic contrast-enhanced imaging. J Comput Assist Tomogr. 2006; 30:7-11.

20. Kim JK, Hong SS, Choi YJ, Park SH, Ahn H, Kim CS, Cho KS. Wash-in rate on the basis of dynamic contrast- enhanced MRI: usefulness for prostate cancer detection and localization. J Magn Reson Imaging. 2005; 22:639-646.

21. Kitajima K, Kaji Y, Fukabori Y, Yoshida K, Suganuma N, Sugimura K. Prostate cancer detection with 3 T MRI: comparison of diffusion-weighted imaging and dynamic contrast-enhanced MRI in combination with T2-weighted imaging. J Magn Reson Imaging. 2010; 31:625-631.

22. Kozlowski P, Chang SD, Meng R, Mädler B, Bell R, Jones EC, Goldenberg SL. Combined prostate diffusion tensor imaging and dynamic contrast enhanced MRI at 3T: quantitative correlation with biopsy. Magn Reson Imaging. 2010; 28:621-628.

23. Reisæter LA, Fütterer JJ, Halvorsen OJ, Nygård Y, Biermann M, Andersen E, Gravdal K, Haukaas S, Monssen JA, Huisman HJ, Akslen LA, Beisland C, Rørvik J. 1.5-T multiparametric MRI using PI-RADS: a region by region analysis to localize the index-tumor of prostate cancer in patients undergoing prostatectomy. Acta Radiol. 2015; 56:500-511.

24. Portalez D, Rollin G, Leandri P, Elman B, Mouly P, Jonca F, Malavaud B. Prospective comparison of T2w-MRI and dynamic-contrast-enhanced MRI, 3D-MR spectroscopic imaging or diffusion-weighted MRI in repeat TRUS-guided biopsies. Eur Radiol. 2010; 20:2781-2790.

25. Puech P, Potiron E, Lemaitre L, Leroy X, Haber GP, Crouzet S, Kamoi K, Villers A. Dynamic contrast-enhancedmagnetic resonance imaging evaluation of intraprostatic prostate cancer: correlation with radical prostatectomy specimens. Urology. 2009; 74:1094-1099.

26. Rosenkrantz AB, Mussi TC, Borofsky MS, Scionti SS, Grasso M, Taneja SS. 3.0 T multiparametric prostate MRI using pelvic phased-array coil: utility for tumor detection prior to biopsy. Urol Oncol. 2013; 31:1430-1435.

27. Rosenkrantz AB, Kim S, Campbell N, Gaing B, Deng FM, Taneja SS. Transition zone prostate cancer: revisiting the role of multiparametric MRI at 3 T. AJR Am J Roentgenol. 2015; 204:W266-W272.

28. Tamada T, Sone T, Jo Y, Yamamoto A, Yamashita T, Egashira N, Imai S, Fukunaga M. Prostate cancer: relationships between post-biopsy hemorrhage and tumor detectability at MR diagnosis. Radiology. 2008; 248:531-539.

29. Tamada T, Sone T, Higashi H, Jo Y, Yamamoto A, Kanki A, Ito K. Prostate cancer detection in patients with total serum prostate-specific antigen levels of 4-10 $\mathrm{ng} / \mathrm{mL}$ : diagnostic efficacy of diffusion-weighted imaging, dynamic contrastenhanced MRI, and T2-weighted imaging. AJR Am J Roentgenol. 2011; 197:664-670.

30. Turkbey B, Mani H, Shah V, Rastinehad AR, Bernardo M, Pohida T, Pang Y, Daar D, Benjamin C, McKinney YL, Trivedi H, Chua C, Bratslavsky G, et.al. Multiparametric 3T prostate magnetic resonance imaging to detect cancer: histopathological correlation using prostatectomy specimens processed in customized magnetic resonance imaging based molds. J Urol. 2011; 186:1818-1824. 
31. Van den Bergh L, Isebaert S, Koole M, Oyen R, Joniau $\mathrm{S}$, Lerut E, Deroose CM, De Keyzer F, Van Poppel H, Haustermans K. Does 11C-choline PET-CT contribute to multiparametric MRI for prostate cancer localisation? Strahlenther Onkol. 2013; 189:789-795.

32. Weidner AM, Michaely HJ, Lemke A, Breitinger L, Wenz F, Marx A, Schoenberg SO, Dinter DJ. Value of multiparametric prostate MRI of the peripheral zone. Z Med Phys. 2011; 21:198-205.

33. Yoshizako T, Wada A, Hayashi T, Uchida K, Sumura M, Uchida N, Kitagaki H, Igawa M. Usefulness of diffusionweighted imaging and dynamic contrast-enhanced magnetic resonance imaging in the diagnosis of prostate transitionzone cancer. Acta Radiol. 2008; 49:1207-1213.

34. Yu JS, Chung JJ, Hong SW, Chung BH, Kim JH, Kim KW. Prostate cancer: added value of subtraction dynamic imaging in 3T magnetic resonance imaging with a phasedarray body coil. Yonsei Med J. 2008; 49:765-774.

35. Zhang X, Quan X, Lu S, Huang F, Yang J, Chan Q, Lin T. The clinical value of dynamic contrast-enhanced magnetic resonance imaging at 3.0T to detect prostate cancer. J Int Med Res. 2014; 42:1077-1084.

36. Ferda J, Kastner J, Hora M, Hes O, Fínek J, Topolčan O, Kreuzberg B. A role of multifactorial evaluation of prostatic 3T MRI in patients with elevated prostatic-specific antigen levels: prospective comparison with ultrasound-guided transrectal biopsy. Anticancer Res. 2013; 33:2791-2795.

37. Vilanova JC, Barceló-Vidal C, Comet J, Boada M, Barceló J, Ferrer J, Albanell J. Usefulness of prebiopsy multifunctional and morphologic MRI combined with free-to-total prostatespecific antigen ratio in the detection of prostate cancer. AJR Am J Roentgenol. 2011; 196:W715-W722.

38. Watanabe H, Kanematsu M, Kondo H, Kako N, Yamamoto N, Yamada T, Goshima S, Hoshi H, Bae KT. Preoperative detection of prostate cancer: a comparison with 11C-choline PET, 18F-fluorodeoxyglucose PET and MR imaging. J Magn Reson Imaging. 2010; 31:1151-1156.

39. Haffner J, Lemaitre L, Puech P, Haber GP, Leroy X, Jones JS, Villers A. Role of magnetic resonance imaging before initial biopsy: comparison of magnetic resonance imagingtargeted and systematic biopsy for significant prostate cancer detection. BJU Int. 2011; 108:E171-178.

40. Weinreb JC, Barentsz JO, Choyke PL, Cornud F, Haider MA, Macura KJ, Margolis D, Schnall MD, Shtern F, Tempany CM, Thoeny HC, Verma S. PI-RADS Prostate Imaging-Reporting and Data System: 2015, Version 2. Eur Urol. 2016; 69:16-40.

41. National Institute for Health and Care Excellence. Prostate cancer: Diagnosis and treatment. Clinical guideline 175. Accessed February 11, 2015. https://www.nice.org.uk/ guidance/cg175/evidence.

42. Bigler SA, Deering RE, Brawer MK. Comparison of microscopic vascularity in benign and malignant prostate tissue. Hum Pathol. 1993; 24:220-226.
43. Engelbrecht MR, Huisman HJ, Laheij RJ, Jager GJ, van Leenders GJ, Hulsbergen-Van De Kaa CA, de la Rosette JJ, Blickman JG, Barentsz JO. Discrimination of prostate cancer from normal peripheral zone and central gland tissue by using dynamic contrast-enhanced MR imaging. Radiology. 2003; 229:248-254.

44. Alonzi R, Padhani AR, Allen C. Dynamic Contrast Enhanced MRI of Prostate Cancer. Medical Radiology. 2007; 63:335-350.

45. Kara T, Akata D, Akyol F, Karcaaltincaba M, Ozmen M. The value of dynamic contrast-enhanced MRI in the detection of recurrent prostate cancer after external beam radiotherapy: correlation with transrectal ultrasound and pathological findings. Diagn Interv Radiol. 2011; 17:38-43.

46. Shimizu T, Nishie A, Ro T, Tajima T, Yamaguchi A, Kono S, Honda H. Prostate cancer detection: the value of performing an MRI before a biopsy. Acta Radiol. 2009; 50:1080-1088.

47. Shukla-Dave A, Hricak H. Role of MRI in prostate cancer detection. NMR Biomed. 2013; 27:16-24.

48. Verma S, Turkbey B, Muradyan N, Rajesh A, Cornud F, Haider MA, Choyke PL, Harisinghani M. Overview of dynamic contrast-enhanced MRI in prostate cancer diagnosis and management. AJR Am J Roentgenol. 2012; 198:1277-1288.

49. Rosenkrantz AB, Mendrinos S, Babb JS, Taneja SS. Prostate cancer foci detected on multiparametric magnetic resonance imaging are histologically distinct from those not detected. J Urol. 2012; 187:2032-2038.

50. Agha M, Eid AF. 3 Tesla MRI surface coil: Is it sensitive for prostatic imaging? Alexandria Journal of Medicine. 2014; 51:111-119.

51. Tofts PS, Brix G, Buckley DL, Evelhoch JL, Henderson E, Knopp MV, Larsson HB, Lee TY, Mayr NA, Parker GJ, Port RE, Taylor J, Weisskoff RM. Estimating kinetic parameters from dynamic contrast-enhanced T(1)-weighted MRI of a diffusable tracer: standardized quantities and symbols. J Magn Reson Imaging. 1999; 10: 223-232.

52. Puech P, Sufana-Iancu A, Renard B, Lemaitre L. Prostate MRI: can we do without DCE sequences in 2013? Diagn Interv Imaging. 2013; 94:1299-1311.

53. Khalifa F, Soliman A, El-Baz A, Abou El-Ghar M, ElDiasty T, Gimel'farb G, Ouseph R, Dwyer AC. Models and methods for analyzing DCE-MRI: A review. Med Phys. 2014; 41:124301.

54. Berman RM, Brown AM, Chang SD, Sankineni S, Kadakia M, Wood BJ, Pinto PA, Choyke PL, Turkbey B. Dce MRI of prostate cancer. Abdom Radiol. 2016; 41:844-853.

55. Haas GP, Sakr WA. Epidemiology of prostate cancer. CA Cancer J Clin. 1997; 47:273-287.

56. Hoeks CM, Schouten MG, Bomers JG, Hoogendoorn SP, Hulsbergen-van de Kaa CA, Hambrock T, Vergunst H, Sedelaar JP, Fütterer JJ, Barentsz JO. Three tesla magnetic resonance-guided prostate biopsy in men with increased prostate-specific antigen and repeated, negative, random, 
systematic, transrectal ultrasound biopsies: detection of clinically significant prostate cancers. Eur Urol. 2012; 62:902-909.

57. Sinnott JA, Rider JR, Carlsson J, Gerke T, Tyekucheva S, Penney KL, Sesso HD, Loda M, Fall K, Stampfer MJ, Mucci LA, Pawitan Y, Andersson SO, Andrén O. Molecular Differences in Transition Zone and Peripheral Zone Prostate Tumors. Carcinogenesis. 2015; 36:632-638.

58. Villers A, Puech P, Leroy X, Biserte J, Fantoni JC, Lemaitre L. Dynamic Contrast-Enhanced MRI for Preoperative Identification of Localised Prostate Cancer. European Urology Supplements. 2007; 6:525-532.

59. Hoeks CM, Hambrock T, Yakar D, Hulsbergen-van de Kaa CA, Feuth T, Witjes JA, Fütterer JJ, Barentsz JO. Transition zone prostate cancer: detection and localization with 3-T multiparametric MR imaging. Radiology. 2013; 266:207-217.

60. Cheikh AB, Girouin N, Colombel M, Maréchal JM, Gelet A, Bissery A, Rabilloud M, Lyonnet D, Rouvière O. Evaluation of T2-weighted and dynamic contrast-enhanced MRI in localizing prostate cancer before repeat biopsy. Eur Radiol. 2009; 19:770-778.

61. Carroll PR, Parsons JK, Andriole G, Bahnson RR, Castle EP, Catalona WJ, Dahl DM, Davis JW, Epstein JI, Etzioni RB, Farrington T, Hemstreet GP, Kawachi MH, et al. NCCN Guidelines Insights: prostate cancer early detection, version 2.2016. J Natl Compr Canc Netw. 2016; 14:509-519.

62. Wolf AM, Wender RC, Etzioni RB, Thompson IM, D'Amico AV, Volk RJ, Brooks DD, Dash C, Guessous I, Andrews K, DeSantis C, Smith RA, American Cancer Society Prostate Cancer Advisory Committee. American cancer society guideline for the early detection of prostate cancer: update. Ca Cancer J Clin. 2010; 60:70-98.

63. Djavan B, Ravery V, Zlotta A, Dobronski P, Dobrovits M, Fakhari M, Seitz C, Susani M, Borkowski A, Boccon-Gibod L, Schulman CC, Marberger M. Prospective evaluation of prostate cancer detected on biopsies 1,2, 3 and 4: when should we stop? J Urol. 2001; 166:1679-1683.

64. Booth A1, Clarke M, Dooley G, Ghersi D, Moher D, Petticrew M, Stewart L. The nuts and bolts of PROSPERO: an international prospective register of systematic reviews. Syst Rev. 2012; 1:2.

65. Leeflang MM, Deeks JJ, Takwoingi Y, Macaskill P. Cochrane diagnostic test accuracy reviews. Syst Rev. 2013; 2:82.

66. Moher D. Preferred reporting items for systematic reviews and meta-analyses: the PRISMA statement. Chin J Integr Med. 2009; 7:889-896.

67. Bucher HC, Guyatt GH, Griffith LE, Walter SD. The results of direct and indirect treatment comparisons in meta-analysis of randomized controlled trials. J Clin Epidemiol.1997; 50:683-691.

68. Whiting PF, Rutjes AW, Westwood ME, Mallett S, Deeks JJ, Reitsma JB, Leeflang MM, Sterne JA, Bossuyt PM. QUADAS-2: a revised tool for the quality assessment of diagnostic accuracy studies. Ann Intern Med. 2011; 155:529-536.

69. Dersimonian R, Laird N. Meta-analysis in clinical trials. Control Clin Trials. 1986; 7:177-88.

70. Macaskill P, Gatsonis C, Deeks JJ, Harbord RM, Takwoingi Y. Chapter10: Analysing and Presenting Results. In: Deeks JJ, Bossuyt PM, Gatsonis C, Editors. Cochrane Handbook for Systematic Reviews of Diagnostic Test Accuracy Version 1.0. The Cochrane Collaboration; 2010.

71. Deeks JJ, Macaskill P, Irwig L. The performance of tests of publication bias and other sample size effects in systematic reviews of diagnostic test accuracy was assessed. J Clin Epidemiol. 2005; 58:882-893.

72. Baker WL, White CM, Cappelleri JC, Kluger J, Coleman CI. Understanding heterogeneity in meta-analysis: the role of meta-regression. Int J Clin Pract. 2009; 63:1426-1434. 